band, das nicht weit über die halbe Länge derselben reicht, versehen, sind lanzettig, nach oben zu zart gewimpert, am Rücken kurz behaart. Staubfäden nur unten wenig und kurz behaart. Die Schuppen stehen ziemlich aufrecht, sind nach oben eiförmig verjüngt und sind nicht ausgerandet, wohl aber höher als breit. Die Fruchtknospe mit Ausnahme der Griffel ist kurz behaart.

Vorko m me $\mathrm{n}$ : Auf der Alpe Promina in Dalmatien (Maly.)

Anmerkung. Schon durch die Form der Rosenblätter, so wie durch die kurze Behaarung ist diese Art von allen übrigen auffallend verschieden. Ich weihe sie Herrn Gustav $\mathbf{S}$ chl eh a n, Director der Steinkohlengruben Dalmatiens, dem Spender werthvoller Unterstützting.

(Fortsetzung folgt.)

\title{
Luzula Forsteri. D. C.
}

Diese nach Dr. M a ly's Enumeratio bisher im ósterreichischen Kaiserstaate in der Lombardie und in Tirol angegebene Planze fand ich im verflossenen Frühjahre in einer ziemlich grossen Anzahl in Wäldern zwischen Kalksburg und Laab, dann im Schönbrunner Parke. Dieser nene Bürger der Flora Wiens ist in dem ausgedehnten Ländercomplexe sicher nicht allein auf diese wenigen Standorte beschränkt, sondern wird ohne Zweifel noch an mehreren anderen Punkten der Monarchie vorkommen, ist aber hier immer mit Luzula vernalis $\mathrm{D}$. C. verwechselt worden. Von diesem letzteren unterscheidet er sich auf den ersten Blick durch die linealen, nicht viel über 1 Linie breiten Wurzelblätter und durch die nach der Blüthe immer aufrecht bleibenden einblüthigen Spirrenäste, welche in diesem Stadium bei vernalis herabgebrochen sind, sonst aber noch durch die über die Kapsel hinausreichenden in eine Stachelspitze endigenden Perigonzipfel, ferner durch das gerade Anhängsel des Samens, endlich durch die gegen die Stachelspitze hin mehr zugespitzte Kapsel, welche bei vernalis unterhalb der Stachelspitze abgestumpft ist.

Wien, den 20. December 1852 . Joh. $0 \mathrm{rtmann}$.

\section{Surrogatpfianzen fitr die Wartofiel.}

Als solche sind unter anderen folgende Pflanzen bekannt geworden: Boussingaultia baselloides H. B. - Apios tuberosa Mö $\mathrm{n} \mathrm{ch}$ (Glycine Apios L. ,Saagabbana"6) - Claytonia virginica. L. die Macca, Occa oder Papa lissa-Ulluirus tuberosus L $\mathrm{oz}$ a n., Ullaco oder Melloco der Peruaner; ferner das Solanum utile Klotsch.

Boussingaultia baselloides, nach E n d li ch er der Familie der Chenopodeae angehörig, kam wie $R$ o us s e 1 o $n$ anführt, $\mathbf{1 8 4 2}$ aus Mexiko und Chili nach Europa. Professor M a g n u zerlegte die Knollen chemisch und fand 16,3 feste Stoffe und $\mathbf{7 3 , 7}$ Wasser, wenig Stärke, aber sehr viel vegetabilischen Schleim. Die Versuche mit dem Anbau ergaben das Resultat, dass die Pflanze leicht au cultiviren sei, dass aber die Knollen des faden Geschmackes wegen kaum für menschliche Nahrung verwendet werden könnten. Kühe, Schafe und Schweine fressen Kraut und Knollen sehr gerne, die 